\title{
Induction of galectin-1 by TLR-dependent PI3K activation enhances epithelial-mesenchymal transition of metastatic ovarian cancer cells
}

\author{
GA BIN PARK $^{1 *}$, YOON HEE CHUNG ${ }^{2 *}$ and DAEJIN KIM ${ }^{3}$ \\ ${ }^{1}$ Department of Biochemistry, Kosin University College of Medicine, Busan 49267; \\ ${ }^{2}$ Department of Anatomy, Chung-Ang University College of Medicine, Seoul 06974; \\ ${ }^{3}$ Department of Anatomy, Inje University College of Medicine, Busan 47392, Republic of Korea
}

Received October 6, 2016; Accepted March 20, 2017

DOI: 10.3892/or.2017.5533

\begin{abstract}
The expression of different toll-like receptors (TLRs) on tumor cells has been associated with disease aggressiveness, treatment resistance, and poor prognosis. The phosphatidylinositol 3-kinase (PI3K)/AKT pathway is considered critical for cancer cell survival and proliferation. Thus, we investigated the effect of TLR-stimulated PI3K activation on the epithelial-to-mesenchymal transition (EMT) of primary (Caov-3) and metastatic (SK-OV-3) epithelial ovarian cancer cell lines in this study. TLR engagement with various ligands promoted the expression of class IA PI3K (p110 $\alpha$, p110 $\beta$, and p1108) and increased the expression of mesenchymal markers (N-cadherin, Slug, Vimentin, Snail, $\alpha$-SMA, and TCF) in SK-OV-3 cells. The migratory activity and secretion of EMT-related cytokines of SK-OV-3 were significantly higher compared to those of Caov-3 after activation with TLR agonist. Although the invasive capacity and production of EMT-related cytokines of LPS-stimulated SK-OV-3 cells were significantly suppressed by all pharmacological inhibitors of the p110 isoform, the Syk/Src-dependent $\mathrm{p} 110 \beta$ isoform prominently attenuated migration activity. In contrast, the production of IL-10 and galectin-1 was mainly affected by the p1108 isoform. Gene silencing of TLR4 and galectin-1 with siRNA decreased the expression of matrix metalloproteinase-2 (MMP2) and MMP9 and reduced mesenchymal markers in LPS-treated SK-OV-3 cells. This study demonstrated that TLR-mediated PI3K activation modulated the invasion and metastasis of ovarian cancer through the production of galectin-1, suggesting
\end{abstract}

Correspondence to: Dr Daejin Kim, Department of Anatomy, Inje University College of Medicine, 75 Bokji-ro, Jin-gu, Busan 47392, Republic of Korea

E-mail:kimdj@inje.ac.kr

${ }^{*}$ Contributed equally

Key words: SK-OV-3, galectin-1, phosphatidylinositol 3-kinase, toll-like receptors, epithelial mesenchymal transition that inhibition of the p110 isoform is a promising therapeutic approach against metastatic ovarian cancer.

\section{Introduction}

The epithelial-to-mesenchymal transition (EMT) is a physiological process that occurs during embryogenesis and wound healing in adults (1). During cancer progression, EMT appears to promote dissemination of cells from the tumor mass, facilitating tissue invasion and metastasis (2). Epithelial ovarian cancer is the most common cause of death from gynecologic tumors worldwide; most cases are detected at advanced stages, which are characterized by diverse metastatic lesions (3).

TLRs are usually expressed in immune cells, such as macrophages and dendritic cells (4). However, in addition to their expression in leukocytes, TLRs are found in multiple tumor types, including in ovarian cancer (5). Stimulation of specific TLRs is associated with carcinogenesis, cancer progression, and site-specific metastasis (6,7). Expression of TLR2, 3, 4, and 5 has been detected on the surface epithelium of normal ovaries and benign and malignant ovarian cancers (8). These data suggest that the TLR-mediated signaling pathway plays a role in EMT and metastasis of ovarian cancer; however, the underlying mechanisms of ovarian cancer metastasis related to TLR stimulation remain unclear.

The phosphatidylinositol 3-kinase (PI3K)/AKT pathway is a critical signaling cascade in the progression and drug resistance of human cancer cells $(9,10)$. TLR4-expressing metastatic colorectal cancer cells activate PI3K/AKT signaling and metastatic capacity after stimulation with lipopolysaccharide (LPS) (11). The phosphatidylinositol-4,5-bisphosphate 3-kinase, catalytic subunit $\alpha(P I K 3 C A)$ gene encodes the catalytic subunit $\mathrm{p} 110 \alpha$ of PI3K class IA, which forms dimers with the regulatory subunit p85 of the same enzyme (12). Over-representation of the PIK3CA gene is one of the most frequently reported abnormalities in ovarian carcinogenesis and is thought to be an early event (13). The p110 $\beta$ isoform is also significantly overexpressed both in ovarian cancer patients and paclitaxel-resistant ovarian cancer cell lines (14). However, whether TLR-mediated PI3K-dependent signaling is correlated with invasive capacity dependent of cancer stage 
Table I. Specific primer sequences used for RT-PCR.

Primers, $5^{\prime} \rightarrow 3^{\prime}$

\begin{tabular}{lll}
\cline { 2 - 3 } Target & \multicolumn{1}{c}{ Sense } & \multicolumn{1}{c}{ Antisense } \\
\hline TLR1 & CGTAAAACTGGAAGCTTGCAAGA & CCTTGGGCCATTCCAAATAAGTCC \\
TLR2 & GGCCAGCAAATTACCTGTGTG & CCAGGTAGGTCTTGGTGTTCA \\
TLR3 & ATTGGGTCTGGGAACATTTCTCTTC & GTGAGATTTAAACATTCCTCTTCGC \\
TLR4 & CTGCAATGGATCAAGGACCA & TCCCACTCCAGGTAAGTGTT \\
TLR5 & CATTGTATGCACTGTCACTC & CCACCACCATGATGAGAGCA \\
TLR6 & TAGGTCTCATGACGAAGGAT & GGCCACTGCAAATAACTCCG \\
TLR7 & AGTGTCTAAAGAACCTGG & CTTGGCCTTACAGAAATG \\
TLR8 & CAGAATAGCAGGCGTAACACATCA & AATGTCACAGGTGCATTCAAAGGG \\
TLR9 & TTATGGACTTCCTGCTGGAGGTGC & CTGCGTTTTGTCGAAGACCA \\
TLR10 & CAATCTAGAGAAGGAAGATGGTTC & GCCCTTATAAACTTGTGAAGGTGT \\
$\beta$-actin & ATCCACGAAACTACCTTCAA & ATCCACACGGAGTACTTGC \\
\hline
\end{tabular}

and which downstream signaling molecules are triggered in TLR-stimulated ovarian cancer cells remain unclear.

In this study, we investigated the difference in TLR-mediated PI3K signaling activity according to ovarian cancer type using primary (Caov-3) and metastatic (SK-OV-3) ovarian cancer cell lines (15). We also examined which catalytic isoforms of class IA PI3Ks (p110 $\alpha$, p110 $\beta$, and p1108) are involved in ovarian cancer metastasis.

\section{Materials and methods}

Cell lines. The human ovarian cancer cell lines Caov-3, OVCAR-3, OV-90, and SK-OV-3 were purchased from the ATCC (Manassas, VA, USA). Caov-3 and OVCAR-3 cells, representing primary cancer, were harvested from human ovarian adenocarcinoma confined to the ovary. OV-90 and SK-OV-3 cells, as representative metastatic cancer, were established from ascites derived from ovarian cancer patients. Caov-3 and OV-90 cells were maintained in DMEM medium (Corning Incorporated, Corning, NY, USA) supplemented with 10\% FBS (RMBIO, Missoula, MT, USA), penicillin, streptomycin, and glutamine at $37^{\circ} \mathrm{C}$ in $5 \% \mathrm{CO}_{2}$. OVCAR-3 and SK-OV-3 cells were maintained in RPMI-1640 medium (Corning Inc.) supplemented with $10 \%$ FBS (RMBIO), penicillin, streptomycin, and glutamine at $37^{\circ} \mathrm{C}$ in $5 \% \mathrm{CO}_{2}$.

Chemicals. LPS (TLR4 ligand) and poly (I:C) (TLR3 ligand) were obtained from Sigma-Aldrich (St. Louis, MO, USA). MALP-2 (TLR2/6 ligand) was purchased from Enzo Life Sciences (Farmingdale, NY, USA). PP1 (Src inhibitor) and Bay 61-3606 (Syk inhibitor) were purchased from Calbiochem (San Diego, CA, USA). A66 (p110 $\alpha$ inhibitor), TGX-221 (p110 $\beta$ inhibitor), CAL-101 (p1108 inhibitor), LY294002 (pan-p110 inhibitor), Bay 80-6946 (p110 $\alpha$ and p110 $\beta$ inhibitor), and pictilisib (p110 $\alpha$ and p110 $\delta$ inhibitor) were obtained from Selleckchem (Houston, TX, USA). Recombinant Gal-1 was purchased from R\&D Systems (Minneapolis, MN, USA).

$R T$-PCR. Total RNA was isolated using an RNeasy Mini kit (Qiagen, Hilden, Germany). RNA was transcribed into cDNA using oligo (dT) primers (Bioneer, Daejeon, Korea) and reverse transcriptase. To investigate the expression of TLR genes in ovarian cancer cells, PCR amplification was performed using specific primer sets (Table I; Bioneer) and Prime Taq Premix (GeNet Bio, Chungnam, Korea). PCR products were analyzed via agarose gel electrophoresis and visualized with ethidium bromide under UV light using the Multiple Gel DOC system (Fujifilm, Tokyo, Japan). Data were analyzed using Image 1.38 software (National Institutes of Health, Bethesda, MD, USA). Experiments were performed in triplicate.

Western blotting. Cells were washed in PBS and lysed in NP-40 buffer (Elpis Biotech, Daejeon, Korea) supplemented with a protease inhibitor cocktail (Sigma-Aldrich). Protein phosphorylation states were preserved through the addition of phosphatase inhibitors (Cocktail II, Sigma-Aldrich) to the NP-40 buffer. Protein concentrations were determined using a BCA assay kit (Pierce, Rockford, IL, USA). Proteins (10 $\mu \mathrm{g} / \mathrm{sample})$ were resolved through SDS-PAGE and then transferred to a nitrocellulose membrane (Millipore Corp., Billerica, MA, USA). Membranes were blocked with 5\% skim milk prior to western blot analysis. Chemiluminescence was detected using an ECL kit (Advansta Corp., Menlo Park, CA, USA) and the Multiple Gel DOC system (Fujifilm). The following primary antibodies were used: E-cadherin, $\mathrm{N}$-cadherin, Slug, Snail, Vimentin, $\alpha$-SMA, TCF8/Zeb1, $\beta$-actin, MMP2, MMP9, Myd88, p110 $\alpha, \mathrm{p} 110 \beta, \mathrm{p} 110 \gamma$, p1108, phosphor-p65, p65, Fyn, phospho-Lyn $\left(\mathrm{Tyr}^{507}\right)$, Lyn, phospho-Src $\left(\mathrm{Tyr}^{416}\right)$, Src, phospho-Syk $\left(\mathrm{Tyr}^{323}\right)$, phospho-Syk (Tyr ${ }^{525 / 526}$ ), and Syk (Cell Signaling Technology, Beverly, MA, USA); $\alpha$-SMA (Bioss, Woburn, MA, USA); and TLR2, TLR3, TLR4, TLR5, TLR6, TLR7, galectin-1, phospho-Fyn $\left(\mathrm{Thr}^{12}\right)$, phospho-PTEN (Ser ${ }^{380} / \mathrm{Thr}^{382 / 383}$ ), and PTEN (Santa Cruz Biotechnology, Santa Cruz, CA, USA).

Small interfering RNA (siRNA) transfection. Experimentally verified human TLR4- and galectin-1-small interfering RNA (siRNA) duplex and negative control-siRNA were obtained from Bioneer. Cells were seeded at a concentration of $1 \times 10^{5}$ per well in a 96-well plate and grown overnight. Cells were then transfected with $200 \mathrm{nM}$ siRNA using Lipofectamine RNAiMAX reagent (Invitrogen, Carlsbad, CA, USA) 
$\mathbf{A}$

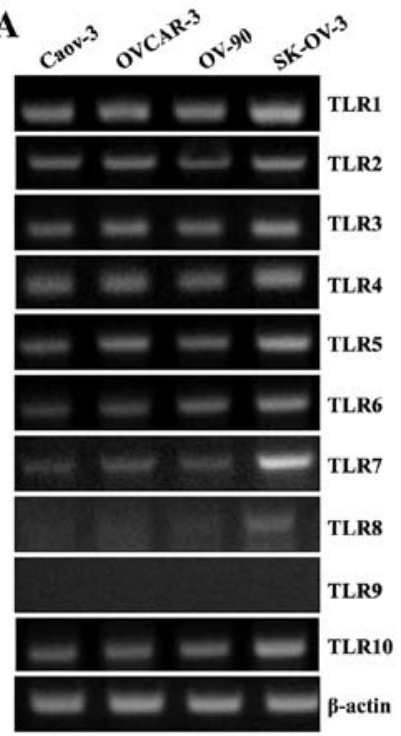

D

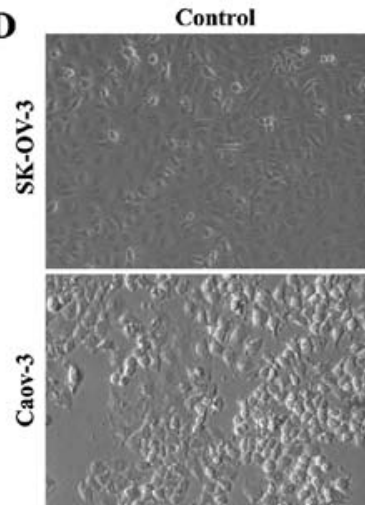

B

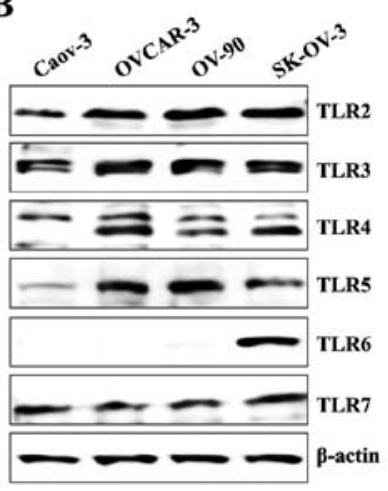

C
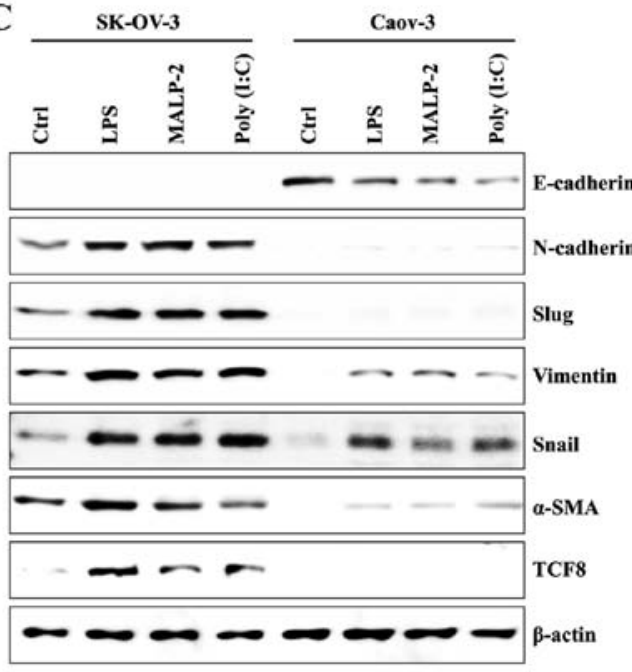

Figure 1. TLR expression and the effects of TLR agonist stimulation on ovarian cancer cells. (A) mRNA expression of TLR1, 2, 3, 4, 5, 6, 7, 8, 9 and 10 in ovarian cancer cells was measured using RT-PCR. (B) Protein expression levels of TLR2, 3, 4, 5, 6 and 7 in ovarian cancer cells were measured using western blotting. $\beta$-actin was used as a loading control. (C) Cells $\left(1.5 \times 10^{5} /\right.$ well) were cultured with and without TLR agonist [poly (I:C), LPS, or MALP2], and levels of EMT markers (E-cadherin, Snail, $\alpha$-SMA, Slug, TCF8, N-cadherin, and Vimentin) were determined using western blot analysis. (D) Comparison of morphologic changes in LPS-stimulated ovarian cancer cells. Photographs were taken at x100 magnification using a digital camera under an inverted phase-contrast microscope (Olympus). (E) The invasiveness of SK-OV-3 cells was enhanced by TLR agonists [poly (I:C), LPS, and MALP2], as determined by a BME cell invasion assay kit. Each value represented the mean \pm standard deviation of three measurements. "p<0.01 (SK-OV-3 vs. Caov-3). Results are representative of three independent experiments.

according to the manufacturer's instructions. Cells were used for further experiments $48 \mathrm{~h}$ after transfection.

Quantification of human cytokines by ELISA. A galectin-1 ELISA assay was performed as previously described (16) using capture Ab (200 ng/ml, $100 \mu 1 /$ well; AF1152; R\&D Systems), detection Ab (100 ng/ml, $100 \mu \mathrm{l} /$ well; BAF1152; R\&D Systems), and standard recombinant galectin-1 (6-500 pg; R\&D Systems). Active TGF- $\beta 1$, TNF- $\alpha$, VEGF, IL-6, IL-8, and IL-10 were quantified using a single cytokine ELISA assay kit (R\&D Systems). Data are expressed as the average of the biological replicates \pm standard deviation (SD).

Invasion assay. Invasion assay was performed using the CultreCoat 96-well Medium BME Cell Invasion assay kit (R\&D Systems) according to the manufacturer's protocol. Cells $\left(2.5 \times 10^{4}\right)$ in serum-free RPMI-1640 or DMEM containing $0.1 \%$ FBS were seeded into the upper chamber, and the lower compartment was filled with RPMI-1640 or DMEM containing $10 \%$ FBS as a chemoattractant. After incubation for $24 \mathrm{~h}$, non-invading cells on the upper membrane surface were removed with a cotton swab. Invaded cells were stained with calcein-AM and quantified using a microplate reader.

Statistical analysis. Data are expressed as the mean \pm standard deviation (SD). Statistical analysis was conducted using one-way analysis of variance. A p-value $<0.05$ was considered statistically significant.

\section{Results}

Stimulation with TLR agonist induces mesenchymal characteristics and invasion activity of $\mathrm{SK}-\mathrm{OV}-3$ cells. Using RT-PCR and western blots, we evaluated the expression of TLRs in both primary (Caov-3 and OVCAR-3) and metastatic (OV-90 and SK-OV-3) ovarian cancer cells. mRNA levels of TLR2, 3, $4,5,6,7$, and 8 in SK-OV-3 cells were significantly elevated compared to other ovarian cancer cells (Fig. 1A). The protein levels of TLR2, 3, 4, 5, and 6 in SK-OV-3 cells were also upregulated compared to Caov-3 cells (Fig. 1B). Next, we investigated 

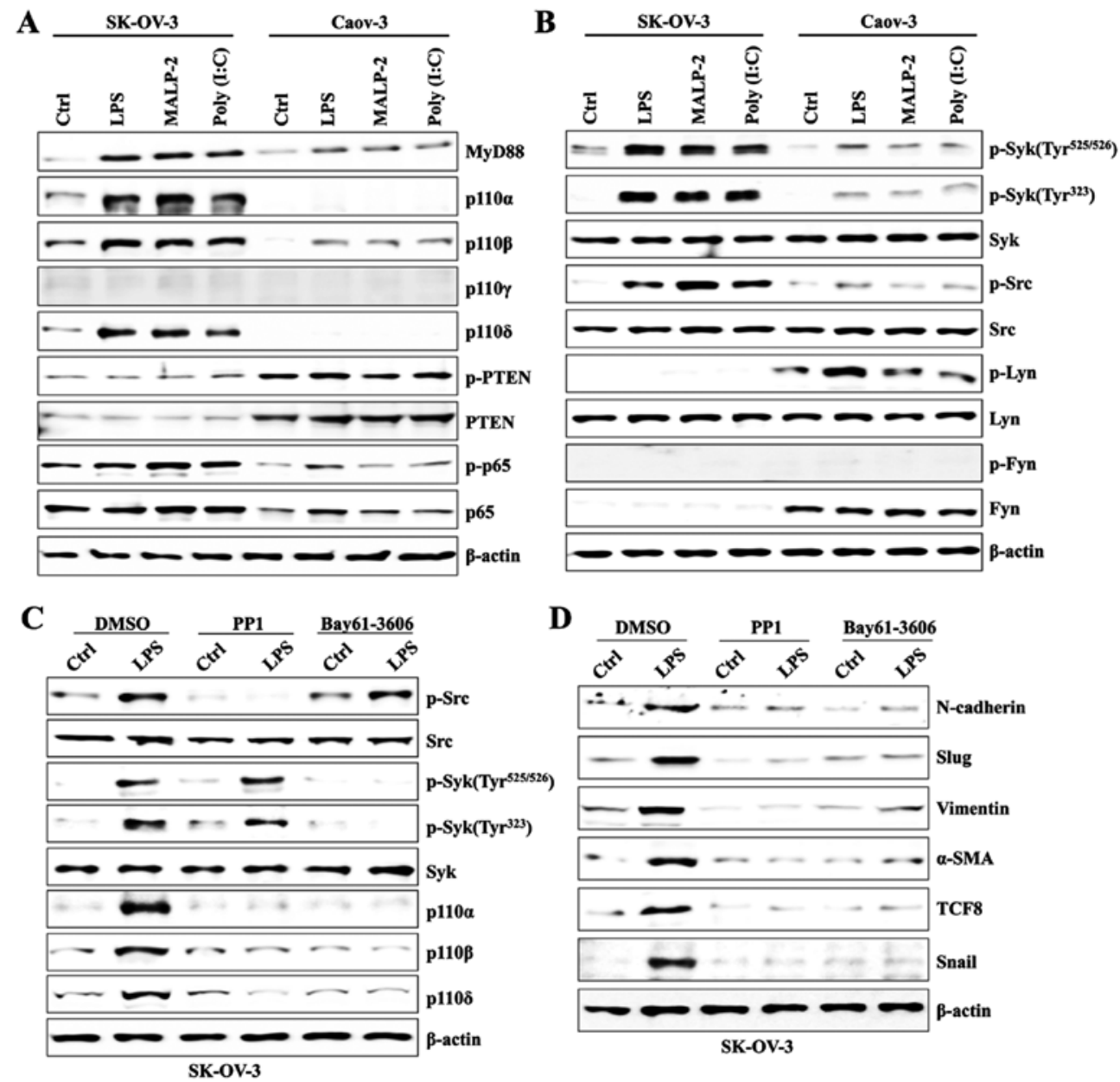

Figure 2. Syk/Src-dependent PI3K activation induces mesenchymal markers in TLR-stimulated SK-OV-3 cells. (A and B) Cells (1.5x10 $/$ well) were cultured with or without TLR3 agonist poly (I:C) $(10 \mu \mathrm{g} / \mathrm{ml})$, TLR4 agonist LPS $(500 \mathrm{ng} / \mathrm{ml})$, or TLR2/6 agonist MALP2 $(1 \mu \mathrm{g} / \mathrm{ml})$ for $24 \mathrm{~h}$. Total cell lysates were immunoblotted with the indicated antibodies. $\beta$-actin was used as the loading control. (C and D) Cells were pretreated with $200 \mathrm{nM}$ of Src inhibitor PP1 or $200 \mathrm{nM}$ of Syk inhibitor BAY-61-3606 for $2 \mathrm{~h}$ and treated with LPS $(500 \mathrm{ng} / \mathrm{ml})$ for $24 \mathrm{~h}$. Total cell lysates were immunoblotted with the indicated antibodies. $\beta$-actin was used as the loading control. Results are representative of three independent experiments.

the effects of TLR2/6 agonist macrophage-activating lipopeptide 2 (MALP-2), TLR4 agonist lipopolysaccharide (LPS), and TLR3 agonist polyinosinic-polycytidylic acid (poly I:C) on changes in EMT-related markers and invasion capacity in primary (Caov-3) and metastatic (SK-OV-3) ovarian cancer cells. TLR activation with various TLR agonists considerably increased mesenchymal characteristics (N-cadherin, Slug, Vimentin, Snail, $\alpha$-SMA, and TCF8) in SK-OV-3 cells compared to Caov-3 cells (Fig. 1C). In addition, LPS stimulation of SK-OV-3 cells increased the population of cells with spindle-shaped morphology compared to LPS-treated Caov-3 cells (Fig. 1D). All TLR agonists significantly increased the migratory capacity of SK-OV-3 cells, but not Caov-3 cells (Fig. 1E). These results suggest that TLR-mediated signaling influences the migratory capacity of metastatic ovarian cancer cell line SK-OV-3.

Syk/Src-dependent PI3K activation induces mesenchymal markers in TLR-stimulated SK-OV-3 cells. Next, we investigated whether TLR stimulation with specific ligands induces activation of PI3K. We also examined Syk- and Src-family tyrosine kinase activity because PI3K signaling is modulated via those tyrosine kinases $(16,17)$. The expression of class IA
PI3Ks (p110 $\alpha, \mathrm{p} 110 \beta$, and p1108) in SK-OV-3 cells was upregulated, as was the expression of MyD88 after stimulation with several TLR ligands; however, MyD88 and the p110 $\beta$ isoform were slightly increased in Caov-3 after engagement with various TLR agonists (Fig. 2A). Although the activity of Syk and Src tyrosine kinases was elevated in LPS-activated SK-OV-3 cells (Fig. 2B), treatment with PP1 (an Src-specific inhibitor) and Bay61-3606 (a Syk-specific inhibitor) efficiently blocked the phosphorylation of tyrosine kinase and the activation of class IA PI3Ks (Fig. 2C). In addition, pharmacological inhibition of Syk and Src tyrosine kinases significantly reduced the expression of EMT-related proteins (Fig. 2D). Our data suggest that Syk/Src-mediated PI3K signal transduction plays an important role in TLR-induced EMT processes of SK-OV-3 cells.

TLR-mediated PI3K activation promotes the secretion of EMT-related cytokines in SK-OV-3 cells. We found that SK-OV-3 cells stimulated by TLR agonist activated class IA PI3Ks (p110 $\alpha, \mathrm{p} 110 \beta$, and $\mathrm{p} 110 \delta$ ). Next, we investigated the effects of specific PI3K inhibition on invasion and secretion of EMT-related cytokines in LPS-stimulated SK-OV-3 cells. Pretreatment with a specific inhibitor (A66, p110 $\alpha$ inhibitor; 


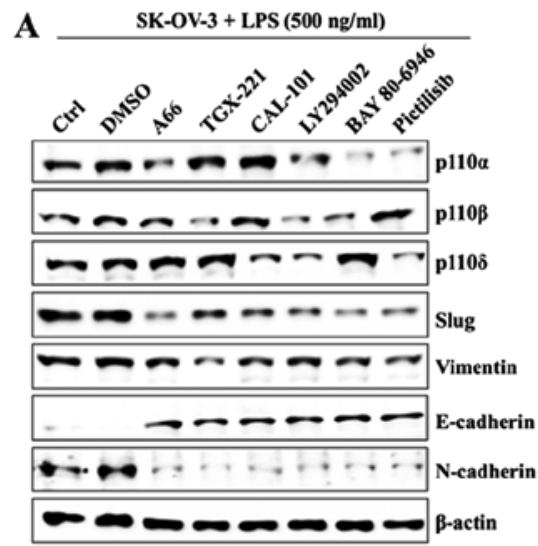

C
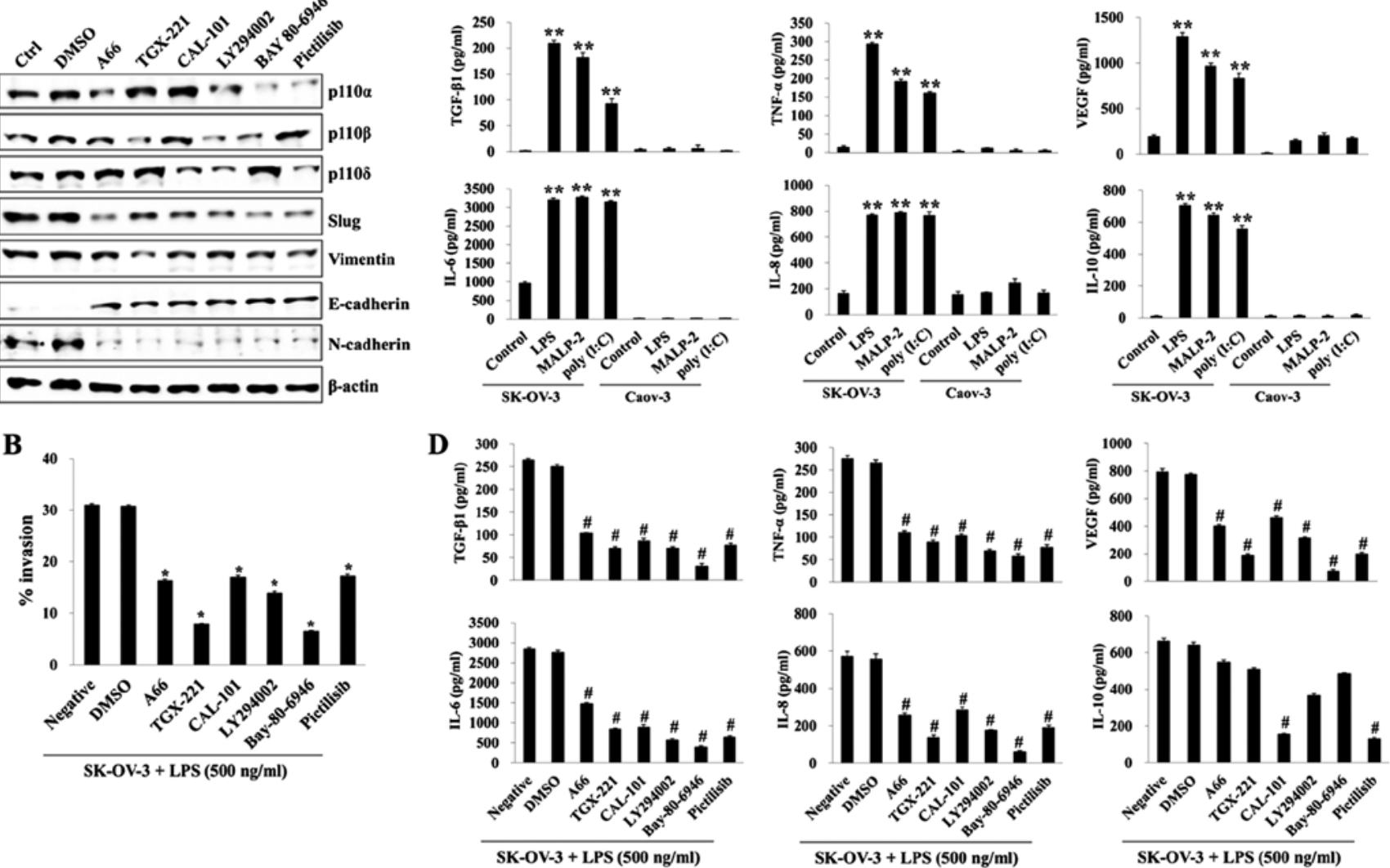

Figure 3. TLR-induced PI3K activation promotes invasion and EMT-related cytokine production in SK-OV-3 cells. SK-OV-3 cells (1.5x10 $/$ well) were pretreated with p110 $\alpha$ inhibitor A66 $(50 \mu \mathrm{M}), \mathrm{p} 110 \beta$ inhibitor TGX-221 $(50 \mu \mathrm{M})$, p1108 inhibitor CAL-101 $(50 \mu \mathrm{M})$, Pan PI3K inhibitor LY294002 (50 $\mu \mathrm{M})$, p110 $\alpha / \beta$ inhibitor Bay80-6946 (200 nM), or p110 $/ \delta$ inhibitor Pictilisib (200 nM) for $2 \mathrm{~h}$. Then cells were treated with LPS (500 ng/ml) for $24 \mathrm{~h}$. (A) Total cell lysates were immunoblotted with the indicated antibodies. $\beta$-actin served as the loading control. (B) The invasiveness of SK-OV-3 cells was inhibited by A66, TGX-221, CAL-101, LY294002, Bay80-6946, or Pictilisib as detected by BME cell invasion assay. Each value represents the mean \pm SD of three measurements. "p $<0.01$ (SK-OV-3+LPS vs. the group treated with each inhibitor). (C) TLR agonists [poly (I:C), LPS, and MALP2] induced the secretion of IL-8, IL-10, VEGF, IL-6, TNF- $\alpha$, or active TGF- $\beta 1$. Cells were seeded onto 6 -well plates $\left(1.5 \times 10^{5} /\right.$ well $)$ and incubated overnight. Culture supernatants were collected at $24 \mathrm{~h}$ after TLR agonist [poly (I:C), LPS, and MALP2] stimulation, and the amounts of IL-8, IL-10, VEGF, IL-6, TNF- $\alpha$, or active TGF- $\beta 1$ were determined by ELISA. Data are representative of two independent experiments and show the mean \pm standard deviation of duplicate determinations. ${ }^{* *} \mathrm{p}<0.001$ (SK-OV-3 vs. Caov-3). (D) SK-OV-3 cells (1.5x10 $/$ well) were pretreated with p110 $\alpha$ inhibitor A66 (50 $\mu \mathrm{M})$, p1 $10 \beta$ inhibitor TGX-221 (50 $\mu \mathrm{M})$, p1108 inhibitor CAL-101 $(50 \mu \mathrm{M})$, Pan PI3K inhibitor LY294002 (50 $\mu \mathrm{M})$, p110 $/ / \beta$ inhibitor Bay80-6946 (200 nM), or p110 $\alpha / \delta$ inhibitor Pictilisib $(200 \mathrm{nM})$ for $2 \mathrm{~h}$, and then the cells were stimulated with LPS for $24 \mathrm{~h}$. The amounts of IL-8, IL-10, VEGF, IL-6, TNF- $\alpha$, or active TGF- $\beta 1$ in culture supernatant were determined by ELISA. Data are representative of two independent experiments and show the mean \pm standard deviation of duplicate measurements. ${ }^{*}$ p $<0.005$ (SK-OV-3+LPS vs. the group treated with each inhibitor). Results are representative of three independent experiments.

TGX-221, p110 $\beta$ inhibitor; CAL-101, p1108 inhibitor; LY294002, p110 $\alpha / \beta / \delta$ inhibitor; Pictilisib, p110 $\alpha / \delta$ inhibitor) for each p110 isoform increased the expression of E-cadherin and decreased the expression of mesenchymal markers in LPS-activated SK-OV-3 cells (Fig. 3A). The migratory activity of LPS-stimulated SK-OV-3 cells was significantly suppressed after treatment with all p110 isoform inhibitors (Fig. 3B). In addition, TGX-221 (p110 $\beta$ inhibitor) and Bay-80-6946 (p110 $\alpha / \beta$ inhibitor) had a prominent influence on invasion attenuation (Fig. 3B). EMT, which is driven by a number of growth factors and cytokines, results in loss of epithelial cell polarities and connections (18). To determine EMT-related cytokine secretion in ovarian cancer cells after TLR stimulation, we compared the concentrations of transforming growth factor $\beta-1$ (TGF- $\beta 1$ ), vascular endothelial growth factor (VEGF), interleukin-6 (IL-6), IL-8, IL-10, and tumor necrosis factor- $\alpha$ (TNF- $\alpha$ ) using a sandwich ELISA method with the culture media in TLR agonist-triggered SK-OV-3 and
Caov- 3 cells. TGF- $\beta 1$ and TNF- $\alpha$, critical cytokines for EMT, were markedly increased in the culture media of SK-OV-3 cells after stimulation with various TLR agonists, whereas those cytokines were barely detectable in the Caov- 3 culture media (Fig. 3C). Other cytokines (VEGF, IL-6, IL-8, and IL-10) associated with invasive activity were also $\sim 3-5$-fold higher than those in non-TLR stimulated SK-OV-3 (Fig. 3C). Although all p110 isoform inhibitors prevented the secretion of EMT-related cytokines, treatment with TGX-221 or Bay-806946 prevented the secretion of EMT-related cytokines most effectively, except for production of IL-10, which was affected by CAL-101 or Pictilisib (Fig. 3D). Our data suggest that TLR-mediated PI3K signaling influences the migratory capacity and secretion of EMT-related cytokines in metastatic ovarian cancer cells.

TLR4-mediated PI3K activation promotes galectin-1 production in LPS-stimulated $S K-O V-3$. Galectin-1 is involved in 

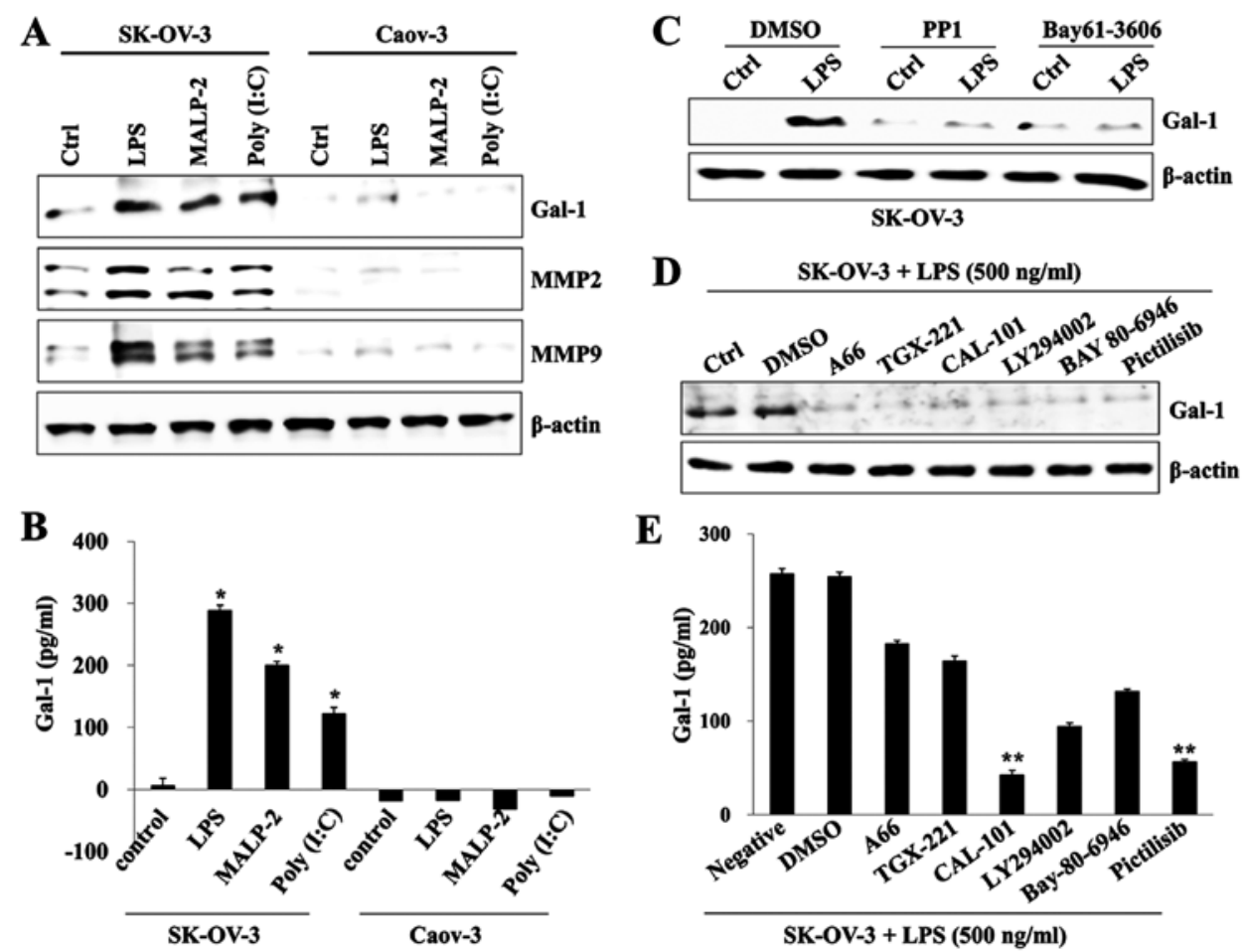

Figure 4. TLR-stimulated PI3K signaling regulates galectin-1 production in SK-OV-3 cells. (A) Cells $\left(1.5 \times 10^{5} /\right.$ well) were cultured with or without TLR3 agonist poly (I:C) $(10 \mu \mathrm{g} / \mathrm{ml})$, TLR4 agonist LPS $(500 \mathrm{ng} / \mathrm{ml})$, or TLR2/6 agonist MALP2 $(1 \mu \mathrm{g} / \mathrm{ml})$ for $24 \mathrm{~h}$. Total cell lysates were immunoblotted with antibody against galectin-1 (Gal-1), MMP2, or MMP9. (B) Culture supernatant was collected $24 \mathrm{~h}$ after TLR agonist (poly (I:C), LPS, and MALP2) stimulation, and the amounts of galectin-1 (Gal-1) were determined by ELISA. Data are representative of two independent experiments and show the mean \pm standard deviation of duplicate measurements. "p<0.001 (SK-OV-3 vs. Caov-3). (C) Cells were pretreated with Src inhibitor PP1 (200 nM) or Syk inhibitor BAY-61-3606 (200 nM) and then treated with LPS $(500 \mathrm{ng} / \mathrm{ml})$ for $24 \mathrm{~h}$. Total cell lysates were immunoblotted with antibody against galectin-1 (Gal-1). $\beta$-actin served as the loading control. (D) Cells were pretreated with p110 $\alpha$ inhibitor A66 (50 $\mu \mathrm{M})$, p110ß inhibitor TGX-221 $(50 \mu \mathrm{M})$, p1108 inhibitor CAL-101 (50 $\mu \mathrm{M})$, Pan PI3K inhibitor LY294002 $(50 \mu \mathrm{M})$, p110 $\alpha / \beta$ inhibitor Bay80-6946 (200 nM), or p110 $\alpha / \delta$ inhibitor Pictilisib (200 nM) for $2 \mathrm{~h}$ and then treated with LPS (500 $\mathrm{ng} / \mathrm{ml})$ for $24 \mathrm{~h}$. Total cell lysates were immunoblotted with antibody against galectin-1 (Gal-1). $\beta$-actin was used as the loading control. (E) Culture supernatant was collected $24 \mathrm{~h}$ after LPS stimulation, and the amount of galectin-1 (Gal-1) was determined by ELISA. Data are representative of two independent experiments and show the mean \pm standard deviation of duplicate measurements. ${ }^{* *} \mathrm{p}<0.005$ (SK-OV-3+LPS vs. the group treated with CAL-101 or Pictilisib).

cancer progression and is associated with a poor prognosis in ovarian cancers (19). We determined the effects of TLR agonists on the production of galectin-1 and investigated the association between activation of PI3K and galectin-1 in ovarian cancer cells. TLR activation using a specific ligand significantly increased the expression of galectin-1, MMP2, and MMP9 in SK-OV-3 cells compared to Caov-3 cells (Fig. 4A and B). Expression in LPS-stimulated SK-OV-3 was suppressed after treatment with an inhibitor of Src (PP1) or Syk (Bay-61-3606) tyrosine kinase (Fig. 4C). Pharmacological inhibition of various p110 isoforms also prevented the expression of galectin-1 (Fig. 4D). In particular, CAL-101 (p1108 inhibitor) and Pictilisib (p110 $\alpha / \delta$ inhibitor) markedly blocked the production of galectin-1 in LPS-activated SK-OV-3 cells compared to the level of other p110 inhibitors (Fig. 4E). Our data suggest that PI3K-dependent galectin-1 production is one of the main pathways of ovarian cancer metastasis after TLR stimulation.

TLR4-mediated galectin-1 production induces EMT of ovarian cancer cells. Next, we investigated whether a TLR4induced signaling pathway regulates galectin-1-mediated migration of ovarian cancer cells. Stimulation with LPS of TLR4-knockdown SK-OV-3 cells failed to increase the production of galectin-1 and the expression of MMP2,
MMP9, and mesenchymal markers (Figs. 5A and B). Treatment with recombinant galectin-1 (r-Gal-1) enhanced the wound healing capacity of TLR4-knockdown SK-OV-3 cells regardless of TLR4 expression or LPS stimulation (Fig. 5C). In addition, targeted inhibition of TLR4 blocked the phosphorylation of Src/Syk kinase and the activation of PI3K in LPS-activated HCT116 cells (Fig. 5D). Finally, we investigated whether TLR4-mediated galectin-1 production affects migration and the expression of mesenchymal markers. Downregulation of galectin-1 with small interfering RNA attenuated the activation of MMP2 and MMP9, and the upregulation of mesenchymal markers in LPS-stimulated SK-OV-3 cells (Fig. 5E). Furthermore, the invasion capacity of galectin-1-knockdown SK-OV-3 cells was profoundly suppressed after stimulation with LPS (Fig. 5F). These results suggest that TLR4-mediated PI3K activation triggers the migratory and invasive capacity of ovarian cancer cells through the production of galectin-1.

\section{Discussion}

TLR activation in leukocytes at the tumor site can stimulate immune cells that can actively fight tumors $(20,21)$; however, their activation can also have tumor-promoting effects (22). Generally, high levels of different TLRs in cancer cells are 

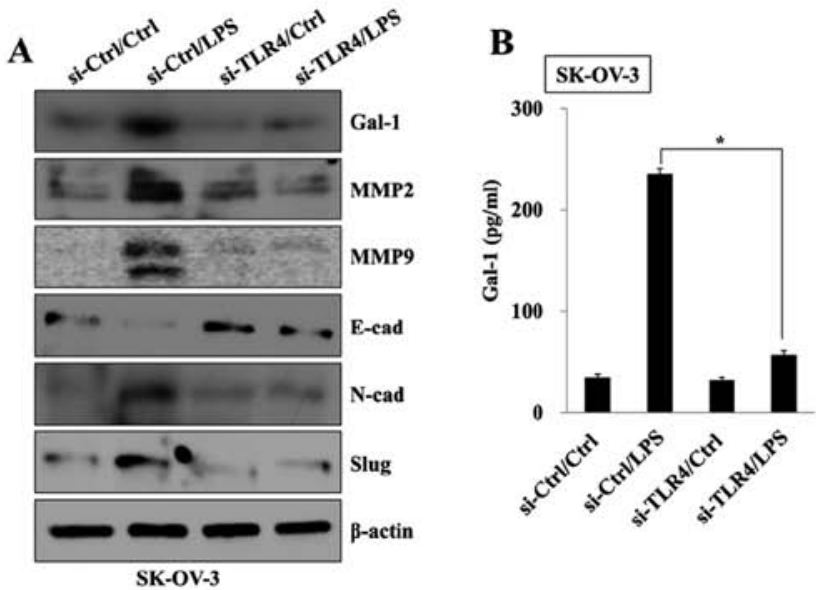

D
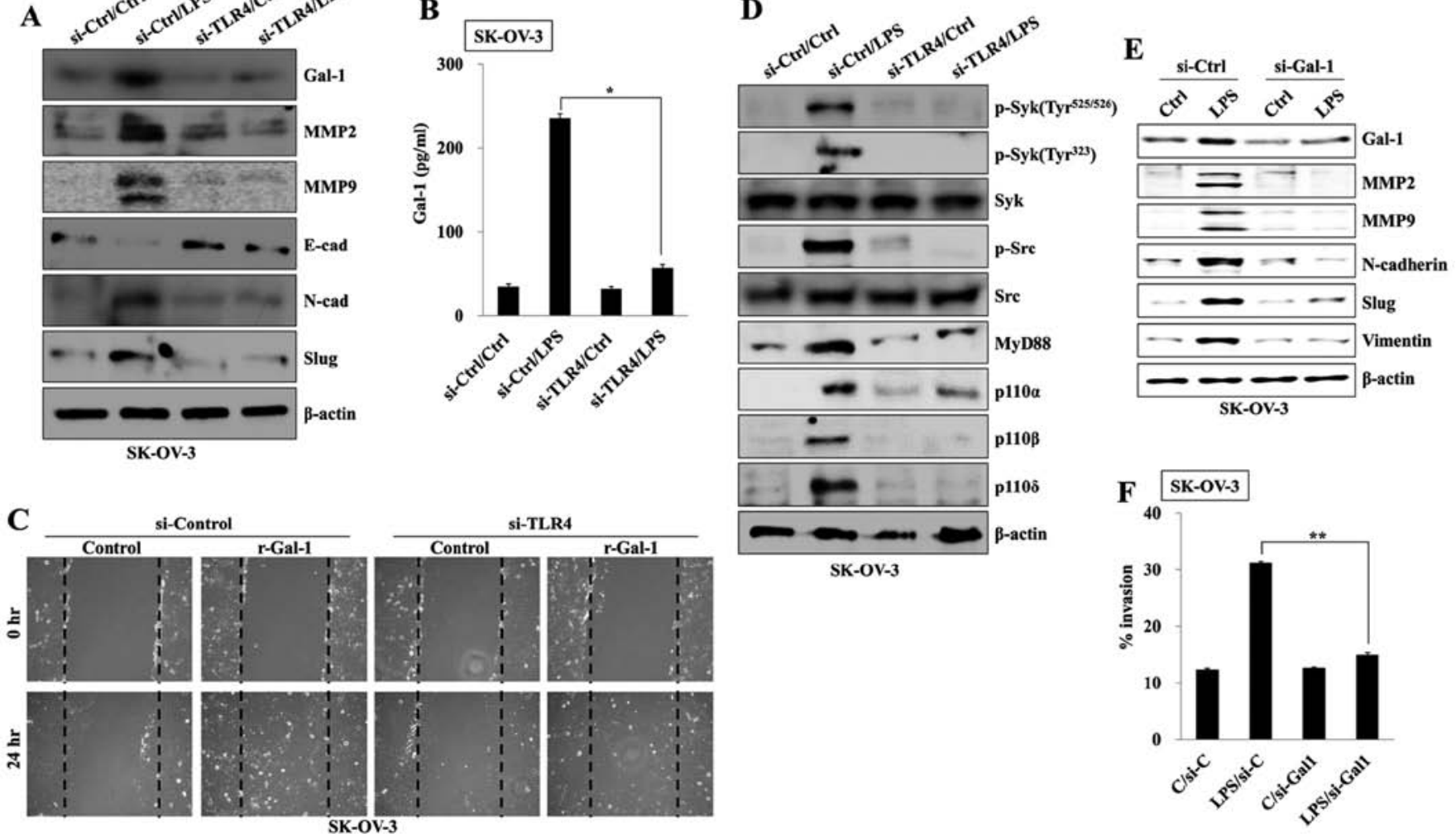

Figure 5. TLR4-mediated galectin-1 production regulates migration and invasion activity in SK-OV-3 cells. TLR4-knockdown SK-OV-3 cells and SK-OV-3 cells transfected with control-siRNA were stimulated with LPS $(500 \mathrm{ng} / \mathrm{ml})$ for $24 \mathrm{~h}$. (A and D) Total cell lysates were collected and immunoblotted with the indicated antibodies. (B) Culture supernatant was collected $24 \mathrm{~h}$ after LPS stimulation, and the amounts of galectin-1 were determined by ELISA. Data are representative of two independent experiments and show the mean \pm standard deviation of duplicate measurements. "p $<0.005$. (C) Comparison of migratory activity of control cells and TLR4-knockdown SK-OV-3 cells after stimulation with recombinant galectin-1 (r-Gal-1). Photographs were taken at x100 magnification using a digital camera under an inverted phase-contrast microscope (Olympus). (E) After transfection with either galectin-1-siRNA or control-siRNA for $48 \mathrm{~h}$, protein levels in SK-OV-3 cells were detected by western blotting with the indicated antibodies. (F) The invasiveness of SK-OV-3 cells was inhibited by galectin-1 silencing, as detected by BME cell invasion assay. Each value represents the mean \pm SD of three determinations. ${ }^{* *} \mathrm{p}<0.05$. Results are representative of three independent experiments.

associated with disease aggressiveness, drug resistance, and poor clinical outcomes $(23,24)$. Administration of paclitaxel with a platinum regimen via an intravenous or intraperitoneal route is the standard chemotherapy for ovarian cancer (25). Unfortunately, paclitaxel stimulates the same signaling pathway via TLR4 to promote secretion of pro-inflammatory cytokines (26). LPS or paclitaxel binding to TLR4 in SK-OV-3 cells promotes IL-6, IL-8, and VEGF production and resistance to drug-induced apoptosis (27). In addition, our study showed that the expression of TLR4 was more prominent in SK-OV-3 than in Caov-3 cells and stimulation with various TLR ligands induced higher expression of mesenchymal markers and invasive activity in SK-OV-3 than in Caov-3 cells. These results suggest that remnant or resistant ovarian cancer cells could use this anticancer drug to promote survival and growth after chemotherapy. Based on these results, TLR levels and expression changes might be critical diagnostic markers in metastatic ovarian cancer.

Stimulation of TLR4 with LPS results in an immediate interaction between PI3K and MyD88, leading to the phosphorylation of AKT (28). Aberrant activation of this pathway has been widely reported in many human cancers, including ovarian cancer (29). Meanwhile, phosphatase and tensin homolog (PTEN), a negative regulator of the AKT signaling pathway, delays the time to progression in ovarian carcinomas and prolongs disease-free survival (30). We observed that Syk/Src-dependent class IA (p110 $\alpha, \mathrm{p} 110 \beta$, and p1108) PI3K activation and expression of mesenchymal markers were significantly increased in TLR-stimulated SK-OV-3 cells; however, the level of PTEN in Caov-3 cells was maintained at a high level after stimulation with TLR agonist. These results suggest that metastatic or invasive ovarian cancer cell lines including SK-OV-3 cells are sensitive to stimulation by TLR ligands, which could lead to the development of aggressive phenotypes.

Although $\mathrm{p} 110 \alpha$ expression is frequently observed in ovarian cancer (13), the levels of p110 $\beta$, but not $\mathrm{p} 110 \alpha$, in ovarian cancer cells are critical factors of drug resistance (14). Other studies also have shown that manifestation of p $110 \alpha$ has no clear relationship with clinical outcome (31). SK-OV-3 cells secrete a higher level of VEGF than Caov-3 cells (32). TLR4-mediated class I PI3K activation in LPS-mediated SK-OV-3 cells is positively correlated with the secretion of EMT-related cytokines in SK-OV-3 cells. Interestingly, selective inhibitor of $\mathrm{p} 110 \alpha$ or $\mathrm{p} 110 \beta$ significantly reduced EMT-related cytokines and targeted co-inhibition of $\mathrm{p} 110 \alpha / \beta$ was the most effective method of reducing EMT-inducing cytokines (TGF- $\beta 1$, TNF- $\alpha$, VEGF, IL- 6 and IL-8), except 
IL-10. IL-10 production was efficiently blocked by CAL-101, a p1108-specific inhibitor, compared to that of LPS-activated SK-OV-3 cells treated with other inhibitors of the p110 isoform. These results suggest that co-administration of anticancer drugs with selective PI3K inhibitors during repeated chemotherapy cycles according to serum cytokine levels in patients could reduce the possibility of metastasis. Furthermore, we need to investigate the signaling pathway to identify precise targets of each condition and need to classify activated p110 isoform- and EMT-associated cytokines in metastatic cancer cells for clinical applications.

Galectin-1 is considered a prototypical galectin and is expressed in many tumor types such as astrocytoma, melanoma, and prostate, thyroid, colon, bladder, and ovarian cancers $(33,34)$. Based on these results, galectin-1 might be a promising candidate for downstream targeting of the TLR/PI3K-mediated signaling pathway in metastatic ovarian cancer. Pharmacological inhibition of PI3K effectively blocked the secretion of galectin-1 in LPS-treated SK-OV-3 cells. Furthermore, knockdown of TLR4 or galectin-1 with siRNA not only suppressed the expression of mesenchymal markers MMP2, and MMP9, but also inhibited invasion activity of LPS-activated SK-OV-3 cells. These results suggest that TLR/PI3K-induced galectin-1 production in ovarian cancer cells is one of the key processes in further invasion and migration.

In conclusion, this study suggests that the TLR/PI3K signaling axis plays a crucial role in facilitating ovarian cancer cell metastasis, and the identification of new molecular targets after TLR engagement is critical for predicting disease progression and clinical outcome.

\section{Acknowledgements}

This study was supported by the Basic Science Research Program of Ministry of Education (NRF-2015R1D1A1A 01056672) and Ministry of Science, ICT and Future Planning (NRF-2015R1C1A2A01053732) through the National Research Foundation (NRF) of Korea.

\section{References}

1. Thiery JP: Epithelial-mesenchymal transitions in tumour progression. Nat Rev Cancer 2: 442-454, 2002.

2. Sabe H: Cancer early dissemination: Cancerous epithelialmesenchymal transdifferentiation and transforming growth factor $\beta$ signalling. J Biochem 149: 633-639, 2011.

3. Vaughan S, Coward JI, Bast RC Jr, Berchuck A, Berek JS, Brenton JD, Coukos G,CrumCC,Drapkin R,Etemadmoghadam D, et al: Rethinking ovarian cancer: Recommendations for improving outcomes. Nat Rev Cancer 11: 719-725, 2011.

4. Akira S, Takeda K and Kaisho T: Toll-like receptors: Critical proteins linking innate and acquired immunity. Nat Immunol 2: 675-680, 2001.

5. Sato Y, Goto Y, Narita N and Hoon DS: Cancer cells expressing toll-like receptors and the tumor microenvironment. Cancer Microenviron 2 (Suppl 1): 205-214, 2009.

6. Chochi K, Ichikura T, Kinoshita M, Majima T, Shinomiya N, Tsujimoto H, Kawabata T, Sugasawa H, Ono S, Seki S, et al: Helicobacter pylori augments growth of gastric cancers via the lipopolysaccharide-toll-like receptor 4 pathway whereas its lipopolysaccharide attenuates antitumor activities of human mononuclear cells. Clin Cancer Res 14: 2909-2917, 2008.

7. Fukata M, Chen A, Vamadevan AS, Cohen J, Breglio K, Krishnareddy S, Hsu D, Xu R, Harpaz N, Dannenberg AJ, et al: Toll-like receptor-4 promotes the development of colitis-associated colorectal tumors. Gastroenterology 133: 1869-1881, 2007.
8. Zhou M, McFarland-Mancini MM, Funk HM, Husseinzadeh N, Mounajjed T and Drew AF: Toll-like receptor expression in normal ovary and ovarian tumors. Cancer Immunol Immunother 58: 1375-1385, 2009.

9. Yu HG, Ai YW, Yu LL, Zhou XD, Liu J, Li JH, Xu XM, Liu S, Chen J, Liu F, et al: Phosphoinositide 3-kinase/Akt pathway plays an important role in chemoresistance of gastric cancer cells against etoposide and doxorubicin induced cell death. Int J Cancer 122: 433-443, 2008.

10. Cantley LC: The phosphoinositide 3-kinase pathway. Science 296: 1655-1657, 2002.

11. Hsu RY, Chan CH, Spicer JD, Rousseau MC, Giannias B, Rousseau S and Ferri LE: LPS-induced TLR4 signaling in human colorectal cancer cells increases betal integrin-mediated cell adhesion and liver metastasis. Cancer Res 71: 1989-1998, 2011.

12. Shayesteh L, Lu Y, Kuo WL, Baldocchi R, Godfrey T, Collins C, Pinkel D, Powell B, Mills GB and Gray JW: PIK3CA is implicated as an oncogene in ovarian cancer. Nat Genet 21: 99-102, 1999.

13. Sham JS, Tang TC, Fang Y, Sun L, Qin LX, Wu QL, Xie D and Guan XY: Recurrent chromosome alterations in primary ovarian carcinoma in Chinese women. Cancer Genet Cytogenet 133: 39-44, 2002.

14. Jeong JY, Kim KS, Moon JS, Song JA, Choi SH, Kim KI, Kim TH and An HJ: Targeted inhibition of phosphatidyl inositol-3-kinase p1 $10 \beta$, but not $\mathrm{p} 110 \alpha$, enhances apoptosis and sensitivity to paclitaxel in chemoresistant ovarian cancers. Apoptosis 18: 509-520, 2013.

15. Vergara D, Merlot B, Lucot JP, Collinet P, Vinatier D, Fournier I and Salzet M: Epithelial-mesenchymal transition in ovarian cancer. Cancer Lett 291: 59-66, 2010.

16. Kurosaki T, Takata M, Yamanashi Y, Inazu T, Taniguchi T, Yamamoto T and Yamamura H: Syk activation by the Src-family tyrosine kinase in the B cell receptor signaling. J Exp Med 179: 1725-1729, 1994.

17. Beitz LO, Fruman DA, Kurosaki T, Cantley LC and Scharenberg AM: SYK is upstream of phosphoinositide 3-kinase in B cell receptor signaling. J Biol Chem 274: 32662-32666, 1999.

18. Guarino M, Rubino B and Ballabio G: The role of epithelialmesenchymal transition in cancer pathology. Pathology 39: 305-318, 2007.

19. Kim HJ, Jeon HK, Cho YJ, Park YA, Choi JJ, Do IG, Song SY, Lee YY, Choi $\mathrm{CH}$, Kim TJ, et al: High galectin-1 expression correlates with poor prognosis and is involved in epithelial ovarian cancer proliferation and invasion. Eur J Cancer 48: 1914-1921, 2012.

20. Scarlett UK, Cubillos-Ruiz JR, Nesbeth YC, Martinez DG, Engle X, Gewirtz AT, Ahonen CL and Conejo-Garcia JR: In situ stimulation of CD40 and Toll-like receptor 3 transforms ovarian cancer-infiltrating dendritic cells from immunosuppressive to immunostimulatory cells. Cancer Res 69: 7329-7337, 2009.

21. Scarlett UK, Rutkowski MR, Rauwerdink AM, Fields J, EscovarFadul X, Baird J, Cubillos-Ruiz JR, Jacobs AC, Gonzalez JL, Weaver J, et al: Ovarian cancer progression is controlled by phenotypic changes in dendritic cells. J Exp Med 209: 495-506, 2012.

22. Liu WT, Jing YY, Yu GF, Han ZP, Yu DD, Fan QM, Ye F, Li R, Gao L, Zhao QD, et al: Toll like receptor 4 facilitates invasion and migration as a cancer stem cell marker in hepatocellular carcinoma. Cancer Lett 358: 136-143, 2015.

23. He W, Liu Q, Wang L, Chen W, Li N and Cao X: TLR4 signaling promotes immune escape of human lung cancer cells by inducing immunosuppressive cytokines and apoptosis resistance. Mol Immunol 44: 2850-2859, 2007.

24. Kelly MG, Alvero AB, Chen R, Silasi DA, Abrahams VM, Chan S, Visintin I, Rutherford T and Mor G: TLR-4 signaling promotes tumor growth and paclitaxel chemoresistance in ovarian cancer. Cancer Res 66: 3859-3868, 2006.

25. Alberts DS, Liu PY, Hannigan EV, O'Toole R, Williams SD, Young JA, Franklin EW, Clarke-Pearson DL, Malviya VK, DuBeshter B, et al: Intraperitoneal cisplatin plus intravenous cyclophosphamide versus intravenous cisplatin plus intravenous cyclophosphamide for stage III ovarian cancer. N Engl J Med 335: 1950-1955, 1996.

26. Byrd-Leifer CA, Block EF, Takeda K, Akira S and Ding A: The role of MyD88 and TLR4 in the LPS-mimetic activity of Taxol. Eur J Immunol 31: 2448-2457, 2001. 
27. Szajnik M, Szczepanski MJ, Czystowska M, Elishaev E, Mandapathil M, Nowak-Markwitz E, Spaczynski M and Whiteside TL: TLR4 signaling induced by lipopolysaccharide or paclitaxel regulates tumor survival and chemoresistance in ovarian cancer. Oncogene 28: 4353-4363, 2009.

28. Laird MH, Rhee SH, Perkins DJ, Medvedev AE, Piao W, Fenton MJ and Vogel SN: TLR4/MyD88/PI3K interactions regulate TLR4 signaling. J Leukoc Biol 85: 966-977, 2009.

29. Kang S, Bader AG and Vogt PK: Phosphatidylinositol 3-kinase mutations identified in human cancer are oncogenic. Proc Nat Acad Sci USA 102: 802-807, 2005.

30. Schöndorf T, Göhring UJ, Roth G, Middel I, Becker M, Moser N, Valter MM and Hoopmann M: Time to progression is dependent on the expression of the tumour suppressor PTEN in ovarian cancer patients. Eur J Clin Invest 33: 256-260, 2003.
31. Wang Y, Kristensen GB, Helland A, Nesland JM, BørresenDale AL and Holm R: Protein expression and prognostic value of genes in the erb-b signaling pathway in advanced ovarian carcinomas. Am J Clin Pathol 124: 392-401, 2005.

32. Sher I, Adham SA, Petrik J and Coomber BL: Autocrine VEGF-A/KDR loop protects epithelial ovarian carcinoma cells from anoikis. Int J Cancer 124: 553-561, 2009.

33. Satelli A and Rao US: Galectin-1 is silenced by promoter hypermethylation and its re-expression induces apoptosis in human colorectal cancer cells. Cancer Lett 301: 38-46, 2011.

34. Ito $\mathrm{K}$ and Ralph SJ: Inhibiting galectin-1 reduces murine lung metastasis with increased CD4(+) and CD8(+) T cells and reduced cancer cell adherence. Clin Exp Metastasis 29: 561-572, 2012. 